\title{
Quantitative genetics of preference and performance on chickpeas in the noctuid moth, Helicoverpa armigera
}

\author{
SC Cotter ${ }^{1,2}$ and OR Edwards ${ }^{2}$ \\ ${ }^{1}$ Department of Biological Sciences, Lancaster Environment Centre, Lancaster University, Lancaster LA1 4YQ, UK; ${ }^{2}$ CSIRO \\ Entomology, Private Bag 5, PO Wembley, WA 6014, Australia
}

If a novel, resistant host-plant genotype arises in the environment, insect populations utilising that host must be able to overcome that resistance in order that they can maintain their ability to feed on that host. The ability to evolve resistance to host-plant defences depends upon additive genetic variation in larval performance and adult host-choice preference. To investigate the potential of a generalist herbivore to respond to a novel resistant host, we estimated the heritability of larval performance in the noctuid moth, Helicoverpa armigera, on a resistant and a susceptible variety of the chickpea, Cicer arietinum, at two different life stages. Heritability estimates were higher for neonates than for third-instar larvae, suggesting that their ability to establish on plants could be key to the evolution of resistance in this species; however, further information regarding the nature of selection in the field would be required to confirm this prediction. There was no genetic correlation between larval performance and oviposition preference, indicating that female moths do not choose the most suitable plant for their offspring. We also found significant genotype by environment interactions for neonates (but not third-instar larvae), suggesting that the larval response to different plant genotypes is stage-specific in this species.

Heredity (2006) 96, 396-402. doi:10.1038/sj.hdy.6800819; published online 29 March 2006

Keywords: biological control; genetic correlation; genotype-by-environment interaction; host-plant resistance; insect; plantinsect interactions

\section{Introduction}

The appearance of a novel, resistant plant genotype in the environment presents a challenge to its herbivores; insect populations utilising that host must be able to evolve resistance in order that they can maintain their ability to feed on that host. To predict how an insect species will respond to the emergence of a new, resistant host genotype, we need to know three things. First, is there additive genetic variation in the insect population for the ability to utilise the resistant host genotype? A number of studies have estimated levels of additive genetic variation in host use in herbivorous insects, the majority of which consider variation in the ability of a generalist herbivore to feed on different host species (Via, 1984a, b; Ward et al, 1993; Carriere and Roitberg, 1994; Fox and Caldwell, 1994; Hawthorne and Via, 1994; Sheck and Gould, 1996; Thompson, 1996; Tucic et al, 1997; Ueno et al, 1997; Bossart, 1998; Hawthorne, 1998; Lazarevic et al, 1998; Gu et al, 2001; Poore and Steinberg, 2001).

Fewer studies examine genetic variation in the ability of a generalist to feed on novel resistant varieties of an existing host, although this is important in terms of the ability of a generalist to maintain its host range. One such study examined variation in the ability of the polyphagous leafminer, Liriomyza trifolii, to feed on a resistant

Correspondence: S Cotter, Department of Biological Sciences, Lancaster Environment Centre, Lancaster University, Lancaster LA1 4YQ, UK.

E-mail: s.cotter@lancaster.ac.uk

Received 10 March 2005; accepted 6 March 2006; published online 29 March 2006 genotype of an existing host, the chrysanthemum (Hawthorne, 1998). Genetic variation for performance on resistant chrysanthemums was identified and 10 generations of selection for resistance in the leafminer resulted in survival in the selected lines matching that found on susceptible chrysanthemum genotypes. Therefore, in this case, the leafminer showed the potential to respond to a novel, resistant genotype of an existing host, thus maintaining its host range.

The second thing we need to know is, if there is additive genetic variation present, at which life stage does this variation manifest itself? For many insect species, the age at which genetic variation in host use becomes apparent could be important. For example, in many lepidopteran species neonates tend to feed on the plant upon which they hatch and so are dependent on their mothers' choice of host, while older larvae are able to move to neighbouring plants, potentially encountering novel plant genotypes (Zalucki et al, 2002). This could result in differing selection pressures at different life stages. Moreover, levels of additive genetic variation can also vary with age or life stage as genes may be differentially expressed, both qualitatively and quantitatively during development (Zhu-Salzman et al, 2003). If the levels of additive genetic variation in performance differ between young and old larvae, then the population-level response to selection could depend more strongly on responses in one life stage over another.

Consequently, the third thing we need to know is whether there is a genetic correlation between offspring performance and adult oviposition preference. For 
example, if there is genetic variation in adult oviposition preference for the resistant host genotype and this is positively genetically correlated with larval performance on the plant, then we would expect a strong response to selection in both of these traits. However, if the adults do not recognise the new, resistant genotype as a host and do not oviposit on it, then the ability of the neonate larvae to develop on the plant may be irrelevant. A number of studies have examined the genetic correlation between preference and performance (Via, 1986; Fox, 1993; Ward et al, 1993; Nylin and Janz, 1996; Tucic et al, 1997; $\mathrm{Gu}$ et al, 2001), but to our knowledge no previous studies have considered age-related effects on additive genetic variation in host use.

Helicoverpa armigera (Hübner) is a highly polyphagous noctuid moth. It is found on a number of agriculturally important species including the chickpea, Cicer arietinum (L.). Chickpea is a self-fertilising species and as such occurs as highly inbred lines. Screening of chickpea germplasm has identified a number of varieties potentially resistant to $H$. armigera (Lateef, 1985). Using the $H$. armigera-chickpea, insect-host relationship as a model system, we address the question of how a polyphagous species might respond to a novel, resistant host genotype by investigating variation in larval performance on, and adult preference for a susceptible and a resistant plant genotype. This variation was then partitioned into additive genetic and residual variance and the genetic correlation across life stages and environments examined.

\section{Methods}

\section{Plants}

Two varieties of the chickpea $C$. arietinum were used in this study: Tyson and ICC506. ICC506 is a variety that has been shown to have high levels of resistance to $H$. armigera in both field and laboratory studies (Lateef, 1985). Early in December 2002, 40 seeds of each variety were sown in commercially available UC Riverside potting soil mix for use in the neonate feeding assays. After 1 week, and 1 month, a further 40 seeds of each variety were sown for use in the third-instar feeding assays and adult oviposition trials, respectively. Thus, a large number of 6-week-old, preflowering plants were available for each assay. All seeds were sacrified prior to sowing and inoculated with rhizobium.

\section{H. armigera culture}

The laboratory culture was founded from larvae collected from northern Western Australia and from several locations on the east coast of Australia. A previous study found that gene flow was high even between distant populations in Australia and that the effective population size was large (Daly and Gregg, 1985). It was on this basis that we decided to collect larvae from sites on the east and west coasts of Australia and outcross them, thus establishing a laboratory colony that encompassed the variation present in the Australian population. To reduce the risk of inbreeding, eggs were collected from more than 200 adults each generation. The colony had been kept in the laboratory for three generations at the beginning of the experiment and was reared at $26^{\circ} \mathrm{C}$ with natural light, a necessary condition for breeding in this species.

\section{Sib analysis}

A full-sib/half-sib design was used to determine heritabilities of feeding performance and adult host preference (Lynch and Walsh, 1998). In all, 15 virgin males were each mated to two virgin females, resulting in 30 families in total. The mated females were then placed in individual containers with access to honey water, filter paper and nappy liner on which to lay eggs. Eggs from each female were collected and allowed to hatch in plastic tubs with access to artificial diet. Immediately upon hatching, 30 neonates from each family were assigned to the neonate assay. Approximately 5 days after hatching, larvae were transferred to individual $25 \mathrm{ml}$ plastic cups. Upon reaching the third instar, 20 larvae per family were assigned to the thirdinstar assay. The remaining larvae were allowed to pupate in the cups and upon emergence moths were assigned to the adult choice assay.

\section{Larval performance assays}

Neonates: From each family, 15 neonates were randomly assigned to the Tyson and 15 to the ICC506 treatment groups. Larvae were then placed, in groups of five, into $200 \mathrm{ml}$ plastic pots containing $10 \mathrm{ml}$ of $10 \mathrm{~g} / 1$ water agar into which the stems of five chickpea leaves were pushed. $H$. armigera moths lay eggs singly, but will lay several eggs on a single leaf ( $S$ Cotter, personal observation). As such, young larvae are likely to encounter each other during feeding. Rearing neonates in groups more closely mimics larval distribution in the field than would rearing in individual containers. Chickpea leaves were collected from Tyson and ICC506 plants immediately prior to testing and randomly distributed among the containers. Larvae were left to feed for 5 days, after which time each surviving larva was weighed. Containers were checked daily to ensure that sufficient leaf material remained. It was not necessary to replace leaf material during the experiment.

Third instars: From each family, 10 third-instar larvae were randomly assigned to the Tyson, and 10 to the ICC506 treatment groups. Larvae were starved for $2 \mathrm{~h}$, weighed and then placed, individually, into $25 \mathrm{ml}$ plastic pots containing $5 \mathrm{ml}$ of $10 \mathrm{~g} / 1$ water agar into which the stems of a single chickpea leaf was pushed. Older $H$. armigera larvae are more solitary and can be cannibalistic and so rearing in individual cups at this stage is necessary (S Cotter, personal observation). Again, chickpea leaves were collected immediately prior to testing and randomly distributed among the containers. After $24 \mathrm{~h}$ of feeding, larvae were weighed a second time to give an estimate of weight gain.

\section{Adult choice assay}

Five female moths from each family were mated and then placed in individual $600 \mathrm{ml}$ containers with two branches of Tyson and two of ICC506 in agar. The branches were matched for size and arranged alternately in a circle around a central feeder. After $24 \mathrm{~h}$, each container was rotated to avoid any positional effects on female choice. Females were left to lay eggs for 2 days, 
after which time the branches were removed and the eggs laid on each counted. Preliminary tests found that the repeatability of female choice using this experimental technique was high $\left(r=0.78 \pm 0.13, \mathrm{MS}_{\text {among females }}=\right.$ $0.193, \mathrm{MS}_{\text {within females }}=0.024, n=2$ preference measurements per female).

\section{Variance components analysis}

Heritability estimates of each trait and genetic correlations between traits were estimated using a multivariate restricted estimate maximum-likelihood (REML) procedure (VCE version 4, Groeneveld and Kovac, 1990; see http://w3.tzv.fal.de/genetik/public_html/). This involved fitting an individual 'animal model' where the phenotype of each individual was separated into additive genetic components of variance plus other random and fixed effects, such that: $\mathbf{y}=X \mathbf{b}+Z \mathbf{a}+\mathbf{e}$, where $\mathbf{y}$ was a vector of phenotypic values, $\mathbf{b}$ and $\mathbf{a}$ were vectors of fixed and random effects, e was a vector of residual values and $X$ and $Z$ were the corresponding design matrices relating records to the appropriate fixed or random effects (Lynch and Walsh, 1998). The phenotypic variance of each trait, $V_{\mathrm{P}}$ is thus described as $V_{\mathrm{P}}=V_{\mathrm{A}}+V_{\mathrm{M}}+V_{\mathrm{R}}$, where $V_{\mathrm{A}}$ is the additive genetic variance, $V_{\mathrm{M}}$ is the variance attributable to maternal effects and $V_{\mathrm{R}}$ is the residual variance, which includes nonadditive sources of genetic variance such as dominance variance or epistatic effects, environmental effects and error variance. All of the estimates for variance due to maternal effects were nonsignificant and so were removed from the models. The effect of cage on the neonate estimates of performance was also nonsignificant and so was removed from the models.

The heritability of each trait was calculated as the ratio of additive genetic variance to phenotypic variance: $h^{2}=V_{\mathrm{A}} / V_{\mathrm{P}}$. Genetic correlations between each pair of traits, $r_{\mathrm{A}}$, were estimated from the genetic covariance estimate between the two traits $\operatorname{Cov}[x, y]$, and the estimate of additive genetic variance for each trait $V_{\mathrm{Ax}}$ and $V_{\mathrm{Ay}}$, where $r_{\mathrm{A}}=\operatorname{Cov}[x, y] /\left[\left(V_{\mathrm{A} x}\right)\left(V_{\mathrm{A} y}\right)\right]^{0.5}$. The VCE program returns SEs for all estimates, the significance of which could then be determined with $t$-tests. As the
REML procedure assumes that the data are normally distributed, larval weight gain data were log-transformed and percentage egg-lay data were angulartransformed prior to analysis to conform to this assumption. The analysis was then repeated with the untransformed data in order to calculate estimates for the coefficients of additive genetic and residual variance $\left(\mathrm{CV}_{\mathrm{A}}\right.$ and $\mathrm{CV}_{\mathrm{R}}$, respectively), where $\mathrm{CV}_{\mathrm{A}}=100\left(V_{\mathrm{A}}\right)^{0.5} / \mathrm{X}$ and $C V_{R}=100\left(V_{R}\right)^{0.5} / X$ and $X$ is the population mean.

\section{Results}

\section{Larval performance and adult oviposition on each chickpea variety}

The effects of plant genotype on larval performance and adult oviposition preference were analysed with linear mixed models using REML in Genstat. We included sire as a random effect, and plant and the interaction between sire and plant as fixed effects. As expected, neonate performance, measured as weight gain over 5 days feeding on chickpea, was significantly higher on the susceptible chickpea Tyson than on the resistant variety ICC506 (Wald statistic $\chi^{2}=106.07, \mathrm{df}=1, P<0.001$, Table 1), although there was no effect of chickpea variety on neonate survival (logistic regression, $\chi^{2}=0.36, \mathrm{df}=1$, $P=0.54)$

Third-instar performance, measured as weight gain over $24 \mathrm{~h}$, was also higher on Tyson, although the effect was much smaller (Wald statistic $\chi^{2}=4.69$, $\mathrm{df}=1$, $P<0.05$; Table 1); there was no mortality in third instars over the course of the feeding test. Despite the fact that the suitability of Tyson for larval development seemed to be higher than that of ICC506, there was no significant difference between the numbers of eggs laid by adult moths on each variety (Wald statistic $\chi^{2}=0.06, \mathrm{df}=1$, $P=0.81$; Table 1 ).

\section{Heritability of larval performance}

All of the heritability estimates for larval performance were highly significant (Table 1). In contrast, the heritability of innate adult host preference was not

Table 1 Trait means and heritability estimates

\begin{tabular}{|c|c|c|c|c|c|c|}
\hline Trait & Untransformed mean & Transformed mean & $\mathrm{N}$ & Heritability & $C V_{A}$ & $C V_{R}$ \\
\hline \multicolumn{7}{|l|}{ Neonate } \\
\hline Tyson & $1.902 \pm 0.083$ & $0.949 \pm 0.023$ & 388 & $0.441 \pm 0.056^{\text {*** }}$ & 70.44 & 72.76 \\
\hline ICC506 & $1.102 \pm 0.040$ & $0.685 \pm 0.016$ & 395 & $0.578 \pm 0.069^{* * *}$ & 59.84 & 57.99 \\
\hline \multicolumn{7}{|l|}{ Third instar } \\
\hline Tyson & $12.86 \pm 0.876$ & $1.166 \pm 0.871$ & 300 & $0.295 \pm 0.056^{* * *}$ & 68.61 & 96.58 \\
\hline ICC506 & $10.48 \pm 0.747$ & $-1.166 \pm 0.745$ & 300 & $0.344 \pm 0.060^{* * *}$ & 72.26 & 100.14 \\
\hline \multicolumn{7}{|l|}{ Adult } \\
\hline Host preference & $0.542 \pm 0.020$ & $0.841 \pm 0.026$ & 116 & $0.053 \pm 0.037^{\mathrm{NS}}$ & 20.47 & 34.60 \\
\hline
\end{tabular}

Neonate performance - untransformed data: weight in $\mathrm{mg}$ after 5 days; transformed data: $\log$ (weight in $\mathrm{mg}$ after 5 days +1 ). Third instar performance - untransformed data: weight gain in $\mathrm{mg}$ over $24 \mathrm{~h}$; transformed data: residuals from the regression of weight after assay on weight before assay. Adult host preference - untransformed data: eggs on Tyson/total eggs on leaves; transformed data: $\arcsin \sqrt{(\text { eggs on Tyson/total eggs on leaves) }}$.

Trait means and heritabilities are given with their SEs. $N$ refers to the number of larvae sampled for each trait; there were 30 families in total. Heritabilities were estimated by partitioning the total variance into additive genetic variance and residual variance. Maternal effects were nonsignificant and so were removed from the model. Coefficients of additive genetic covariation $\left(\mathrm{CV}_{\mathrm{A}}\right)$ for transformed data are meaningless (Houle, 1992); therefore, $\mathrm{CV}_{\mathrm{A}}$ and $\mathrm{CV}_{\mathrm{R}}$ were calculated for the untransformed data. $\mathrm{NS}=$ nonsignificant.

${ }^{N S} P>0.05 ;{ }^{* * *} P<0.001$. 
Table 2 Genetic correlations between traits

\begin{tabular}{|c|c|c|c|c|}
\hline & Neonate - ICC506 & Third instar - Tyson & Third instar - ICC506 & Adult host preference \\
\hline Neonate - Tyson & $0.198^{\mathrm{NS}}$ & $0.014^{\mathrm{NS}}$ & $0.061^{\mathrm{NS}}$ & $-0.108^{\mathrm{NS}}$ \\
\hline Neonate - ICC506 & & $0.393^{* *}$ & $0.517^{* * *}$ & $0.325^{\mathrm{NS}}$ \\
\hline Third instar - Tyson & & & $0.945^{* * *}$ & $0.128^{\mathrm{NS}}$ \\
\hline Third instar - ICC506 & & & & $0.038^{\mathrm{NS}}$ \\
\hline
\end{tabular}

Neonate performance, transformed data: $\log$ (weight in $\mathrm{mg}$ after 5 days +1 ). Third-instar performance, transformed data: residuals from the regression of weight after assay on weight before assay. Adult host preference, transformed data: arcsin $\sqrt{(\text { eggs on Tyson/total eggs on leaves) }}$. Values show genetic correlations as estimated by VCE. There were 30 families in total. Significance levels were determined with $t$-tests $\mathrm{NS}=$ nonsignificant.

${ }^{\mathrm{NS}} P<0.05 ;{ }^{* *} P<0.01 ;{ }^{* * *} P<0.001$.

significant, although the $\mathrm{CV}_{\mathrm{A}}$ values calculated using the untransformed data suggest that there is additive genetic variation present in this trait.

The estimates for the heritability of neonate performance on each host plant were higher than the respective third-instar estimates, although this was marginally nonsignificant for heritability of performance on Tyson (Tyson, $h_{\text {neonate }}^{2}=0.441 \pm 0.050, h_{3}^{2}$ rd instar $=0.295 \pm 0.056$, $t_{28}=1.95, \quad P=0.061 ; \quad$ ICC506, $\quad h_{\text {neonate }}^{2}=0.578 \pm 0.069$, $\left.h_{3 \text { rd instar }}^{2}=0.344 \pm 0.060, t_{28}=2.56, P<0.05\right)$. There was a trend for the heritability estimates of performance on ICC506 to be higher than on Tyson, but this was not significant (Table 1). An examination of the $C_{A}$ and $C V_{R}$ calculated for each trait show that there are similar levels of additive genetic variation present in all the measures of performance, but that the levels of residual variance are higher for third-instar performance (Table 1).

\section{Genetic correlations across life stages}

While there was a significant positive genetic correlation between neonate and third-instar performance on ICC506 $\left(r_{\mathrm{A}}=0.517 \pm 0.122, t_{13}=4.24, P<0.001\right.$; Table 2$)$, there was no comparable correlation across life stages for larvae feeding on Tyson $\left(r_{\mathrm{A}}=0.014 \pm 0.142, t_{13}=0.10\right.$, $P>0.05$; Table 2).

Trait variation and the environment

Trait variation across environments can be examined in two ways. Firstly, variable trait expression can be regarded as the trait itself and variation partitioned into that explained by the genotype and that explained by the environment. A significant genotype-by-environment interaction shows that genotypes perform relatively differently in each environment. The second approach considers trait expression in each environment as a different trait and examines the genetic covariance between them; a genetic correlation significantly lower than 1 indicates that the ranking of genotypes differs across environments (Lynch and Walsh, 1998).

Using the first approach, there was a significant genotype-by-environment interaction for neonate performance (Wald statistic $\chi^{2}=68.87, \mathrm{df}=28, \quad P<0.001$; Figure 1a) but not for third-instar performance (Wald statistic $\chi^{2}=12.84, \mathrm{df}=28, P=0.99$; Figure $1 \mathrm{~b}$ ). The second approach confirms this result. The genetic correlation across environments for the neonates was significantly lower than $1\left(r_{\mathrm{A}}=0.198+0.130, t_{13}=-6.17\right.$, $P<0.001$; Table 2), whereas the correlation across environments for the third-instar larvae was not sig-
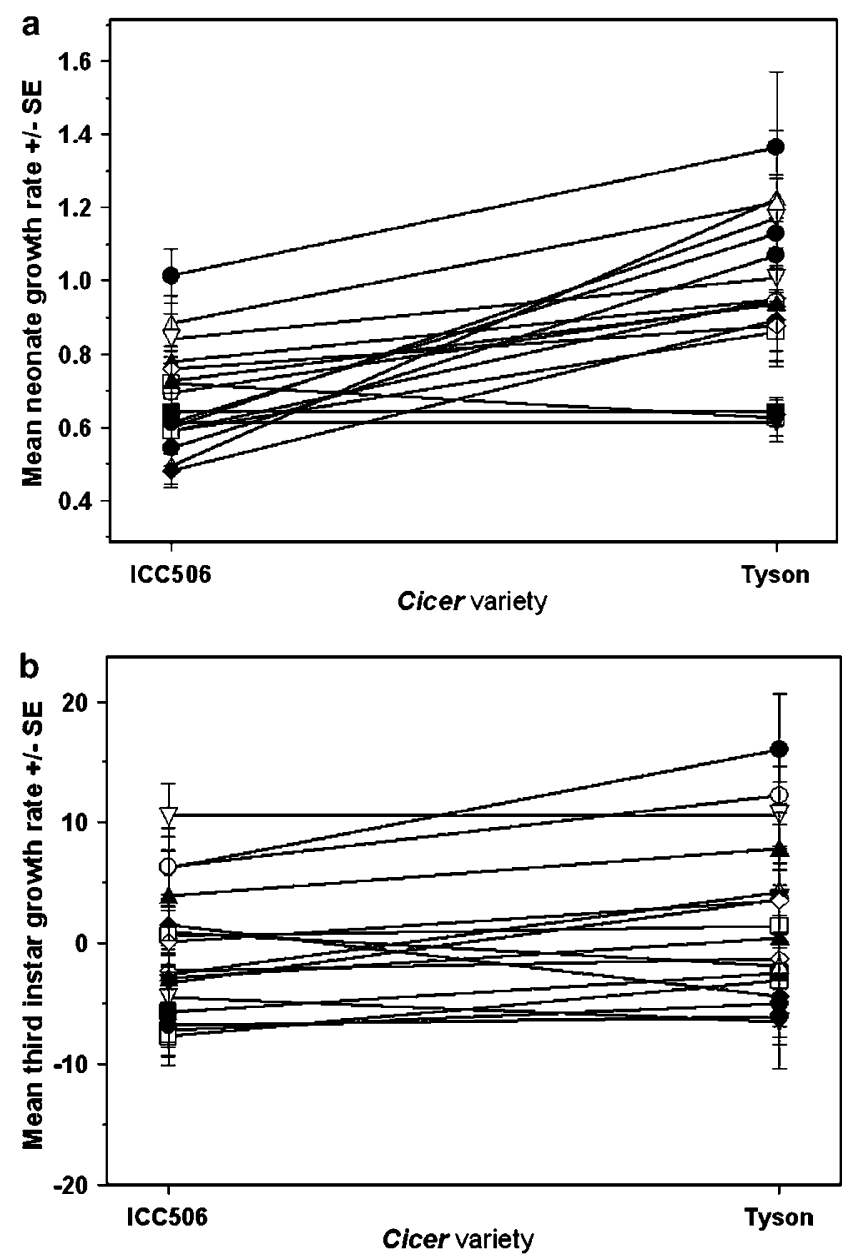

Figure 1 Genotype-by-environment interactions for each life stage. Family means $\pm S E$ using the transformed data are plotted for each chickpea variety for neonates (a) and for third instars (b). Nonparallel lines are an indication of a genotype-by-environment interaction. Neonate growth rate is measured as log (weight in $\mathrm{mg}$ after 5 days +1 ). Third-instar growth rate is measured as the residuals from the regression of weight after assay on weight before assay.

nificantly different from $1\left(r_{\mathrm{A}}=0.945+0.049, t_{13}=-1.12\right.$, $P=0.28$; Table 2).

\section{Discussion}

To predict how a generalist will respond to the emergence of a new, resistant host genotype in the 
environment, we need to know three things: is there additive genetic variation for the ability to utilise the resistant host genotype, at which life stage does this variation manifest itself and is there a genetic correlation between offspring performance and adult oviposition preference?

In this study, we found moderate levels of additive genetic variation in the ability to utilise a susceptible chickpea and a novel, resistant chickpea genotype in the $H$. armigera population. It has been argued that $\mathrm{CV}_{\mathrm{A}}$ rather than heritability should be used as an indicator of evolvability (Houle, 1992). Heritability is the measure of evolvability in the standard deviation (SD)-standardised version of the breeders equation (Lynch and Walsh, 1998); however, Hereford et al (2004) suggest that standardising by the SD is inappropriate as the standardisation factor is itself a function of the additive genetic variance. Alternatively, standardising by the trait mean results in the measure of evolvability being the $C V_{A}$. As such, if the mean-standardised strength of selection is equal for all measured traits, the $\mathrm{CV}_{\mathrm{A}}$ would be the best predictor of the response to selection, whereas if the SD-standardised strength of selection is equal for all measured traits, the heritability would be the best predictor of the response to selection.

Interestingly, the heritability of performance was higher for neonates than third-instar larvae, suggesting that the SD-standardised response to selection for performance on a plant should be stronger in neonates than in older larvae. In contrast, the $\mathrm{CV}_{\mathrm{A}}$ values calculated from the untransformed data were very similar across all groups, suggesting that the meanstandardised response to selection would be similar at both life stages. It seems likely that the probability an individual will survive to reproduce in the field would be associated with its absolute growth rate rather than with its growth rate relative to other individuals. As such, it is not possible to predict which form of standardised selection would be more comparable to that acting in the field. Therefore, in order to predict the response to selection in the field, further information regarding the nature of selection at each life stage would be required.

On the resistant chickpea genotype, ICC506, there was a strong positive genetic correlation in larval performance across the life stages. If the basis of this correlation is pleiotropy rather than linkage disequilibrium, then the genes controlling the trait at each life stage were largely the same. Conversely, on the susceptible genotype, Tyson, there was no genetic correlation in performance across life stages. It is interesting to note that there was also a strong positive genetic correlation between neonate performance on ICC506 and third-instar performance on Tyson. This poses interesting questions regarding the genes controlling larval performance on each host.

A possible explanation for the pattern of genetic correlations in larval performance could be due to constitutive versus induced resistance to plant defence mechanisms at the different life stages. Plants use a number of resistance mechanisms that can affect insect feeding, including physical factors such as leaf toughness or trichome density, or chemical factors such as toxic allelochemicals and proteinase inhibitors. When encountering chemical defences, insects can respond in kind.
For example, insects can detoxify allelochemicals via the inducible cytochrome $P 450$ monoxygenase system (Berenbaum, 1991; Berenbaum et al, 1992; Rose et al, 1992; Hung et al, 1995; Scott et al, 1998; Harrison et al, 2001; Li et al, 2002), or produce or upregulate alternative proteases that are not susceptible to inhibition, or that can digest the proteinase inhibitors present in the diet (Broadway, 1996, 1997; Wu et al, 1997; Patankar et al, 1999, 2001; Moon et al, 2004).

The specific mechanism of resistance responsible for the differences between Tyson and ICC506 is unknown; however, the evidence to date suggests that resistance in chickpeas is primarily due to acid exudates on the leaf surface (Lateef, 1985) or isoflavonoids (Simmonds and Stevenson, 2001); it is therefore likely to involve some kind of secondary compound to which the feeding larva is forced to respond. Neonates have few fat reserves and establishment on a plant is critical to survival. In these circumstances, it would be beneficial for a detoxification system to be an induced response, switched on only when necessary. In other words, neonates placed on the resistant host, ICC506, faced with secondary compounds would respond by switching on genes responsible for the production of detoxifying enzymes or insensitive proteases, while neonates on Tyson, the more palatable host, would not.

Third-instar larvae with greater fat reserves may have these genes switched on as a form of constitutive resistance, or alternately, the threshold level of gut function disruption at which these genes are switched on may be much lower than for neonates. This scenario could result in genes being switched on in all larvae tested, except for the neonates on Tyson. Further studies are necessary to determine the secondary compounds responsible for resistance in ICC506 and the mechanisms of detoxification used by $H$. armigera in response to these compounds in the diet.

In contrast to performance, adult oviposition preference was not heritable and there was no overall preference for the susceptible chickpea, Tyson (at least under the experimental protocol employed here). It may be that the females were unable to discriminate between the two varieties of chickpea. Both lines appear to be identical, and while there may be differences in secondary compounds between the lines, these may not be detectable prior to ingestion. As such, there may be no chemical cues available to a female to indicate that one line is less suitable for her offspring than another. A lack of genetic correlation between oviposition preference and offspring performance, while counterintuitive, is not unusual even at the level of species, (Thompson, 1988 and references therein; Fox, 1993; Nylin and Janz, 1996; Gu et al, 2001), and as such, it may be that the lack of genetic correlation in this case is accurate.

Previous studies have suggested that adult host preference is strongly influenced by factors such as plant abundance and experience with a particular host (Papaj and Rausher, 1987; Cunningham et al, 1998, 1999; Cunningham and West, 2001). Whether the lack of preference for either genotype of chickpea found here is due to the inability to discriminate between the two or due to a lack of experience with either host, it seems likely that females encountering a large patch of host plants, as would occur in an agricultural situation, would 
oviposit regardless of the genotype present. Under these circumstances, it seems likely that there would be strong selection acting on neonates for improved performance and that the response to such selection would be rapid. Future studies should focus on the strength of selection at each life stage to further examine the potential for insect adaptation to resistant hosts in the field.

\section{Acknowledgements}

We thank Kate Detchon and Louisa Bell for technical assistance, and James Ridsdill-Smith, Leigh Simmons, Ken Wilson and anonymous reviewers whose comments greatly improved this manuscript. This work was funded by the Grains Research and Development Council.

\section{References}

Berenbaum MR (1991). Comparative processing of allelochemicals in the Papilionidae (Lepidoptera). Arch Insect Biochem Physiol 17: 213-221.

Berenbaum MR, Cohen MB, Schuler MA (1992). CytochromeP450 monooxygenase genes in oligophagous Lepidoptera. ACS Symp Ser 505: 114-124.

Bossart JL (1998). Genetic architecture of host use in a widely distributed, polyphagous butterfly (Lepidoptera: Papilionidae): adaptive inferences based on comparison of spatiotemporal populations. Biol J Linn Soc 65: 279-300.

Broadway RM (1996). Dietary proteinase inhibitors alter complement of midgut proteases. Arch Insect Biochem Physiol 32: 39-53.

Broadway RM (1997). Dietary regulation of serine proteinases that are resistant to serine proteinase inhibitors. J Insect Physiol 43: 855-874.

Carriere Y, Roitberg BD (1994). Trade-offs in responses to host plants within a population of a generalist herbivore, Choristoneura rosaceana. Entomol Exp Appl 72: 173-180.

Cunningham JP, Jallow MFA, Wright DJ, Zalucki MP (1998). Learning in host selection in Helicoverpa armigera (Hubner) (Lepidoptera: Noctuidae). Anim Behav 55: 227-234.

Cunningham JP, West SA (2001). Host selection in phytophagous insects: a new explanation for learning in adults. Oikos 95: 537-543.

Cunningham JP, Zalucki MP, West SA (1999). Learning in Helicoverpa armigera (Lepidoptera: Noctuidae): a new look at the behaviour and control of a polyphagous pest. Bull Entomol Res 89: 201-207.

Daly JC, Gregg P (1985). Genetic variation in Heliothis in Australia: species identification and gene flow in the two pest species $H$. armigera (Hübner) and $H$. punctigera. Wallengren (Lepidoptera: Noctuidae) 75: 169-184.

Fox CW (1993). A quantitative genetic analysis of oviposition preference and larval performance on 2 hosts in the Bruchid beetle, Callosobruchus maculatus. Evolution 47: 166-175.

Fox CW, Caldwell RL (1994). Host-associated fitness trade-offs do not limit the evolution of diet breadth in the small milkweed bug Lygaeus kalmii (Hemiptera, Lygaeidae). Oecologia 97: 382-389.

Groeneveld E, Kovac M (1990). A generalized computing procedure for setting up and solving mixed linear-models. J Dairy Sci 73: 513-531.

Gu H, Cao A, Walter GH (2001). Host selection and utilisation of Sonchus oleraceus (Asteraceae) by Helicoverpa armigera (Lepidoptera: Noctuidae): a genetic analysis. Ann Appl Biol 138: 293-299.

Harrison TL, Zangerl AR, Schuler MA, Berenbaum MR (2001). Developmental variation in cytochrome $P 450$ expression in Papilio polyxenes in response to xanthotoxin, a host plant allelochemical. Arch Insect Biochem Physiol 48: 179-189.
Hawthorne D (1998). Predicting insect adaptation to a resistant crop. J Econ Entomol 91: 565-571.

Hawthorne DJ, Via S (1994). Variation in performance on 2 grape cultivars within and among populations of grape Phylloxera from wild and cultivated habitats. Entomol Exp Appl 70: 63-76.

Hereford J, Hansen TF, Houle D (2004). Comparing strengths of directional selection: how strong is strong? Evolution 58: 2133-2143.

Houle D (1992). Comparing evolvability and variability of quantitative traits. Genetics 130: 195-204.

Hung CF, Harrison TL, Berenbaum MR, Schuler MA (1995). CYP6B3 - a 2nd furanocoumarin-inducible cytochrome- $P 450$ expressed in Papilio polyxenes. Insect Mol Biol 4: 149-160.

Lateef SS (1985). Gram pod borer (Heliothis armigera) (Hub.) resistance in chickpeas. Agric Ecosyst Environ 14: 95-102.

Lazarevic J, Peric-Mataruga V, Ivanovic J, Andjelkovic M (1998). Host plant effects on the genetic variation and correlations in the individual performance of the Gypsy moth. Funct Ecol 12: 141-148.

Li XC, Berenbaum MR, Schuler MA (2002). Plant allelochemicals differentially regulate Helicoverpa zea cytochrome $P 450$ genes. Insect Mol Biol 11: 343-351.

Lynch M, Walsh B (1998). Genetics and Analysis of Quantitative Traits. Sinauer Associates: Sunderland, MA.

Moon J, Salzman RA, Ahn J, Koiwa H, Zhu-Salzman K (2004). Transcriptional regulation in cowpea bruchid guts during adaptation to a plant defence protease inhibitor. Insect Mol Biol 13: 283-291.

Nylin S, Janz N (1996). Host plant preferences in the comma butterfly (Polygonia c-album): do parents and offspring agree? Ecoscience 3: 285-289.

Papaj DR, Rausher MD (1987). Genetic differences and phenotypic plasticity as causes of variation in oviposition preference in Battus philenor. Oecologia 74: 24-30.

Patankar AG, Giri AP, Harsulkar AM, Sainani MN, Deshpande VV, Ranjekar PK et al (2001). Complexity in specificities and expression of Helicoverpa armigera gut proteinases explains polyphagous nature of the insect pest. Insect Biochem Mol Biol 31: 453-464.

Patankar AG, Harsulkar AM, Giri AP, Gupta VS, Sainani MN, Ranjekar PK et al (1999). Diversity in inhibitors of trypsin and Helicoverpa armigera gut proteinases in chickpea (Cicer arietinum) and its wild relatives. Theor Appl Genet 99: 719-726.

Poore AGB, Steinberg PD (2001). Host plant adaptation in an herbivorous marine amphipod: genetic potential not realized in field populations. Evolution 55: 68-80.

Rose RL, Gould F, Levi P, Konno T, Hodgson E (1992). Resistance to plant allelochemicals in Heliothis virescens (Fabricius). ACS Symp Ser 505: 137-148.

Scott JG, Liu N, Wen ZM (1998). Insect cytochromes P450: diversity, insecticide resistance and tolerance to plant toxins. Comp Biochem Physiol C 121: 147-155.

Sheck AL, Gould F (1996). The genetic basis of differences in growth and behavior of specialist and generalist herbivore species: selection on hybrids of Heliothis virescens and Heliothis subflexa (Lepidoptera). Evolution 50: 831-841.

Simmonds MSJ, Stevenson PC (2001). Effects of isoflavonoids from Cicer on larvae of Helicoverpa armigera. J Chem Ecol 27: 965-977.

Thompson JN (1988). Evolutionary ecology of the relationship between oviposition preference and performance of offspring in phytophagous insects. Entomol Exp Appl 47: 3-14.

Thompson JN (1996). Trade-offs in larval performance on normal and novel hosts. Entomol Exp Appl 80: 133-139.

Tucic N, Mikuljanac S, Stojkovic O (1997). Genetic variation and covariation among life history traits in populations of 
Acanthoscelides obtectus maintained on different hosts. Entomol Exp Appl 85: 247-256.

Ueno H, Fujiyama N, Katakura H (1997). Genetic basis for different host use in Epilachna pustulosa, a herbivorous ladybird beetle. Heredity 78: 277-283.

Via S (1984a). The quantitative genetics of polyphagy in an insect herbivore. I. Genotype-environment interaction in larval performance on different host plant species. Evolution 38: 881-895.

Via S (1984b). The quantitative genetics of polyphagy in an insect herbivore. II. Genetic correlations in larval performance within and among host plants. Evolution 38: 896-905.

Via S (1986). Genetic covariance between oviposition preference and larval performance in an insect herbivore. Evolution 40: 778-785.
Ward KE, Hayes JL, Navasero RC, Hardee DD (1993). Genetic variability in oviposition preference among and within populations of the cotton Bollworm (Lepidoptera: Noctuidae). Ann Entomol Soc Am 86: 103-110.

Wu YR, Llewellyn D, Mathews A, Dennis ES (1997). Adaptation of Helicoverpa armigera (Lepidoptera: Noctuidae) to a proteinase inhibitor expressed in transgenic Tobacco. Mol Breed 3: 371-380.

Zalucki MP, Clarke AR, Malcolm SB (2002). Ecology and behavior of first instar larval Lepidoptera. Ann Rev Entomol 47: 361-393.

Zhu-Salzman K, Koiwa H, Salzman RA, Shade RE, Ahn JE (2003). Cowpea bruchid Callosobruchus maculatus uses a three-component strategy to overcome a plant defensive cysteine protease inhibitor. Insect Mol Biol 12: 135-145. 